\title{
Écriture et topographie dans le voyage romantique : la figure du
} gouffre

\section{Claude Reichler}

\section{Citer ce document / Cite this document :}

Reichler Claude. Écriture et topographie dans le voyage romantique : la figure du gouffre. In: Romantisme, 1990, n69. Procès d'écritures Hugo-Vittez. pp. 3-13;

doi : 10.3406/roman.1990.5665

http://www.persee.fr/doc/roman_0048-8593_1990_num_20_69_5665

Document généré le 26/05/2016 


\section{Ecriture et topographie dans le voyage romantique : la figure du gouffre}

«Plus de route, de précipices, de gorge ni de ciel : rien que du blanc à songer ", écrit Rimbaud le 17 novembre 1878. Il est à Gênes, attendant un passage pour l'Egypte. Ensuite, ce sera Chypre, puis l'Abyssinie. Il en a assez de la littérature, celui qui s'en va vers l'Orient des comptoirs, des chantiers, de tous les trafics. Pourtant, en décrivant ainsi le passage du Gothard dans une lettre à sa famille, il réactive une fois de plus un stéréotype romantique. Lui qui pense, ayant franchi les Alpes, en avoir fini avec les gouffres, avait-il lu le poème de Baudelaire paru dix ans auparavant dans la troisième édition des Fleurs du mal :

Pascal avait son gouffe, avec lui se mouvant.

- Hélas ! tout est abîme, - action, désir, rêve,

Parole !

Peut-être avait-il lu aussi cette relation d'un voyage en Valais que Gautier avait fait paraître cette même année dans les livraisons d'octobre du Moniteur universel:

Une vapeur légère, transparente d'abord comme une gaze, mais qui bientôt s'épaissit, ne laissait apercevoir la profondeur de l'abîme que comme à travers un rêve. Rien de plus singulier que ce gouffre blanc, laiteux, opalin, qui ressemblait à l'infini sans forme et sans couleur.

[...] Il nous semblait suivre au flanc d'un pic sortant du fond de l'abîme et se prolongeant jusqu'au ciel une étroite corniche sur l'infini, au-dessus des ténèbres blanches et du vaporeux non-être ${ }^{1}$.

Effet de brume qui gomme les arêtes et les à-pic jusqu'à domestiquer le gouffre affreux, lui donner l'allure onirique et ouatée d'une berceuse métaphysique. Le sentiment d'abîme qui habite le romantique n'a rien perdu de son intensité dans ces tardifs réemplois d'un thème connu. Lisant Gautier, on pense au célèbre tableau de Friedrich, peint vers 1818, Le Voyageur au-dessus de la mer de nuages. Le voyageur est seul, à l'avant-plan, occupant le centre de la toile. Il a le dos tourné, le vent fouette sa chevelure, il est tout entier au spectacle des cimes et des chaînes qui se déploient devant lui par étagements dans les lointains. La jambe gauche est en avant, fléchie légèrement, la droite est en arrière, comme s'il venait de faire le dernier pas de son ascension, à l'extrême bord du rocher où s'est posé son pied. Le rocher forme une masse sombre, tourmentée et fissurće, qui s'avance en triangle depuis le bas du tableau, qu'il occupe tout entier, jusqu'à sa pointe où se tient le voyageur. Ce rocher est un promontoire; il est une proue, un vaisseau des altitudes qui porte le voyageur vers l'avant, et vers le haut. De la mer de 
nuages que l'homme domine émergent quelques rocs, quelques végétations : juste assez pour donner le sentiment que, en-dessous des volutes et des mèches cotonneuses qui remplissent le centre du paysage, s'ouvre un abîme. Ce que le tableau fait voir est beaucoup moins important que ce qu'il donne à pressentir, à ressentir. Dressé sur son étrave minérale, ami des vents, le voyageur aventure son regard par dessus les précipices. Il est le passager des abîmes.

\section{Contempler}

Se déroulant selon des moments obligés, le voyage obéit à une poétique à laquelle les écrivains sont particulièrement attentifs. Ces « moments » appartiennent à la fois au monde de l'expérience (au voyage effectué) et au voyage écrit, voire aux voyages lus. Ils sont les lieux communs de cette poétique, les figures où le monde et l'écriture se nouent à un sujet. Ce sont des lieux (ou des instants) de configuration : le monde, le texte et le sujet prennent forme ensemble. Lorsqu'un écrivain fait de son voyage un texte, il amplifie et dramatise certaines figures : la préparation, le départ, le déplacement, le franchissement, la découverte, la déception, l'errance, le retour..., deviennent des emblèmes porteurs d'un savoir vécu et chargés d'une puissante efficacité symbolique ${ }^{2}$.

L'abîme dramatise le franchissement. Il est un obstacle vide, une barrière paradoxale, creusée devant les pieds du voyageur. Par la marche, le saut, le vol ou la navigation - fût-ce même par le regard - il faut franchir l'abîme, ou interrompre le voyage. Construisons cette figure du récit de voyage en tenant compte du retentissement subjectal et de la réinterprétation historique. On sait que le XVIIIe siècle connaît une révolution dans la perception des paysages, notamment par la découverte de la montagne, tenue jusqu'alors pour un effrayant désert. On sait qu'avec Albrecht von Haller et les voyageurs anglais, Rousseau est pour beaucoup dans cette vogue nouvelle. Son texte le plus célèbre est sans doute la lettre du Valais, dans La Nouvelle Hélö̈se, mais les Confessions ne manquent pas non plus de passages mille fois imités, où les Alpes et leurs précipices constituent le cadre d'une expérience des gouffres. Voici, au livre IV, Jean-Jacques revenant à Chambéry et passant le défilé de Chailles, qu'on appelait « le Pas-de-l'Echelle », ou « les Echelles ». Le chemin, qui est sur la route de l'Italie par le Mont Cenis, a été souvent décrit, entre autres par Chateaubriand et Stendhal. Il court au flanc du rocher, taillé de main d'homme et bordé d'un parapet : le torrent gronde tout au fond de la gorge :

Bien appuyé sur le parapet $j$ 'avançais le nez, et je restais là des heures entières, entrevoyant de temps en temps cette écume et cette eau bleue dont j'entendais le mugissement à travers les cris des corbeaux et des oiseaux de proie qui volaient de roche en roche et de broussaille en broussaille à cent toises au-dessous de moi. Dans les endroits où la pente était assez unie et la broussaile assez claire pour laisser passer des cailloux, j’en allais chercher au loin d'aussi gros que je les pouvais porter, je les rassemblais sur le parapet en pile ; puis les lançant l'un après l'autre, je me délectais à les voir rouler, bondir et voler en mille éclats avant que d'atteindre le fond du précipice.

Outre le plaisir du voyage et le ton enjoué du récit, il y a dans ce texte les éléments d'une sorte de charte du sentiment d'abîme, qui seront si souvent pré- 
sents dans les textes romantiques, de Byron à Flaubert et au-delà. Et d'abord le regard fasciné, scrutant l'abîme, qui se mue parfois en contemplation : on a vu Friedrich et Gautier dissimuler le gouffre pour l'éprouver plus intensément. Hugo s'installe au bord des précipices et y plonge son regard ; il cherche un point de vue dominant pour déployer le grand lyrisme des antithèses et des métaphores. Le voici au Rigi, masse conique isolée située au-dessus de Lucerne :

J'étais sur une étroite esplanade de roche et de gazon accrochée comme un balcon au mur démesuré du Rigi. J'avais devant moi dans tout leur développement le Bürgen, le Buochserhorn et le Pilate ; sous moi, à une profondeur immense, le lac de Lucerne, morcelé par les anses et les golfes, où se miraient ces faces de géant comme dans un miroir cassé...

Dans une anfractuosité du rocher, assis les jambes pendantes sur une grosse pierre, un idiot, un goitreux, à corps grêle et à face énorme, riait d'un rire stupide, le visage en plein soleil, et regardait au hasard devant lui. $O$ abîme ! Les Alpes étaient le spectacle, le spectateur était un crétin ${ }^{3}$.

Le goût des abîmes que manifeste Hugo trouve dans le voyage des Alpes un espace idéal. Un imaginaire déployé comme une palette produit, à l'aide des clichés alpestres (le voyage en Suisse est déjà largement une suite de chromos en 1839), un paysage abyssal, vaste, et pourtant toujours organisé. Il n'est pas jusqu'au fameux « crétin des Alpes » qui ne trouve place dans le jeu des reflets et des oppositions, où il devient tour à tour un repoussoir et un double du moi, dans lequel Hugo contemple métaphoriquement le mystère insondable de l'homme. Le gouffre ouvert sous les pieds du voyageur s'ouvre aussi dans son âme. De même, le lac où se mirent les montagnes (décrites ici en termes anthropomorphes: les faces de géants) est une image de la psyché hugolienne. Cette exploitation systématique d'une relation « en miroir » entre l'homme et le paysage requiert pour explication une théorie que la notation d'Amiel à propos de Rousseau (« le paysage est un état de l'âme ») ne satisfait pas. Il ne s'agit pas tant d'une relation sentimentale entre l'homme et la nature, que d'une substitution réciproque établie dans l'esprit du sujet : d'une relation que, remettant à plus tard le développement spéculatif de cette notion, j'appellerai de symbolisation.

Rouler, sombrer, s'abîmer

Hugo creuse le gouffre et campe sur ses bords, alors que d'autres s'y précipitent ou rêvent d'y rouler. Le texte de Rousseau dégageait très nettement ce moment de la chute dans le regard fasciné jeté vers le fond, et surtout dans le jeu qui l'accompagne et l'accomplit. Jean-Jacques lance des pierres et les suit des yeux ; il déjoue la peur du vide en délégant des objets qui représentent le moi et le déchargent. Activité et geste symboliques qui satisfont la curiosité et évitent l'angoisse. Gautier, on le sait déjà, module admirablement les jeux du vertige, les visions de profondeur et les plongées imaginaires. Il sait rendre légère et euphorique l'inquiétante attirance, donner au fantasme de chute un ton d'ironique maîtrise. Mais il possède aussi une gamme sombre. Voici un texte où se retrouvent tous les éléments présents chez Rousseau : mais les rocs et les arbres sont doués d'agressivité, ils tranchent et broient ; l'eau claire et bruissante est devenue un sombre Cocyte ; l'angoisse et l'évocation d'une chute réclle ont remplacé le jeu : 
Quelle chute affreuse, comme on rebondirait hideusement fracassé, vivant peutêtre encore, d'arbre en arbre, de roche en roche, pêle-mêle avec les débris de la voiture et les cadavres de chevaux, jusqu'à ce qu'on arrivât tout sanglant à cette noire profondeur où bouillonne le torrent, qui vous reprendrait et vous tordrait dans ses tourbillons! Ces pensées vous traversent l'esprit rapides comme l'éclair, surtout lorsque la route surplombe quelque précipice à pic dont rien ne vous sépare, pas même le plus mince parapet ${ }^{4}$.

Ces effets d'imagination dynamique auxquels Gautier se plaît et qui conviennent si bien aux descriptions de paysages alpestres, Dumas les utilise pour intensifier ses récits. Dans ses Impressions de voyage en Suisse (publiées de 1833 à 1837), il fait montre d'une véritable passion pour les gouffres et les chutes. Dumas se retrouve sans cesse au bord de précipices, de grottes, de déchirures profondes, de parois à pic. De l'alpiniste au chasseur de chamois, en passant par le paysan fauchant son pré au bord du vide, il ne manque aucun cliché. L'un des mieux dramatisés est le passage de la Gemmi : pressé de découvrir le paysage, Dumas arrive presque courant sur le sentier qui contourne une cabane et se trouve tout à coup au sommet d'une paroi verticale ; six cents mètres plus bas, quelques minces fumées, quelques toits en pente : le village. Pas de jet de pierres ici, pas de brumes opportunes, pas de contemplation sereine, mais un sentiment brutal et insurmontable : l'attrait du gouffre, le besoin de s'y jeter, l'aspiration de la chute, le vertige ! Dans cette impression violente, qui scelle un pacte entre l'homme et le gouffre, se révèle une intimité avec le vide, un sentiment pathétique d'Icare sombrant. Faisant allusion au personnage de Byron qui se précipite du haut de la montagne, on pourrait le nommer, comme aurait fait Gaston Bachelard, le complexe de Manfred...

Dans cette expérience, le voyageur romantique reconnaît au gouffre un caractère satanique. Il ne le franchit pas, il s'y abîme. Les enfers et leur paysage torturé font partie des valeurs symboliques associées aux précipices. Dans la gravure pittoresque du XIXe siècle, Le Pont du Diable est cent fois représenté : arc léger jeté sur un abîme entre les roches inhumaines, surplombant l'eau bouillonnante et les pierres aiguês. Infimes passants dans l'immensité naturelle, des voyageurs le traversent, à pied ou à dos de mulet, tels des marcheurs immobiles figés là pour illustrer le destin de l'homme, être du passage, du passager, voué à l'angoisse de la chute. Dumas joue de cette référence infernale dans un épisode de son Voyage, lors de la visite aux Bains de Pfeffers où, comme à la Gemmi, lui est surpris pr la brusque découverte de l'abîme :

On ouvrit la porte, et la chose changea. Cette porte donnait sur une espèce d'abîme au fond duquel roulait la Tamina, entraînant avec elle des rochers, qu'elle arrondit en les frottant sur son lit de marbre noir...

Nous continuâmes de suivre ce dangereux chemin, et nous nous engageâmes sous cette gorge infernale, entendant gronder sous nos pieds le torrent, que nous n'osions regarder de peur des vertiges. [...] Tout à coup mon guide me fit remarquer deux ombres qui, pareilles à Orphée et à Eurydice, semblaient remonter de l'enfer ; elles venaient à nous du fond de la caverne et chaque fois qu'elles passaient sous un de ces soupiraux, elles s'illuminaient d'un jour blafard qui n'avait rien de vivant ${ }^{5}$. 
D'une manière éminemment significative, l'homme qui remonte ainsi du sombre creux est un peintre; de même, la comparaison avec Orphée n'est pas due au hasard. C'est à l'artiste romantique que revient la mission de passer de l'autre côté, après avoir touché le fond du gouffre... C'est à lui de témoigner, de raconter. La descente dans l'abîme est aussi une épreuve initiatique. Dans ses Mémoires d'Outre-Tombe, Chateaubriand raconte sa visite au Niagara dans les termes de l'expérience romantique du gouffre. Il avait déjà décrit la cataracte dans l'Essai sur les révolutions et dans Atala, en 1797 et en 1802 : la thématique de l'abîme était présente, mais la description était traitée comme un tableau vu par un spectateur qui embrasserait différents points de vue en même temps. Dans les Mémoires, dont cette partie est rédigée en 1822 et revue dans les années quarante, Chateaubriand utilise la perspective des voyageurs, qui met en scène le sujet comme partie de son expérience. Comme s'il avait lu Dumas ou Hugo, il décrit moins l'abîme que l'effet que celui-ci produit sur le voyageur. Coutumier de l'hyperbole, il va jusqu'à raconter une prétendue chute dans le gouffre : voulant voir la cataracte d'en bas, il s'accroche à une liane et descend le long du rocher ; la liane manque, le voilà suspendu par une main au-dessus du vide. Il tombe, et par miracle une saillie le reçoit pour conserver au XIXe siècle son plus grand écrivain... «J'étais à un demi-pied de l'abîme et je n'y avais pas roulé » ${ }^{6}$. Les Sauvages, qu'il appelle à son secours comme les divinités d'en haut, le tireront de là et le soigneront, achevant, après ce baptême du gouffre, l'initiation que Chateaubriand était venu chercher dans le Nouveau Monde.

\section{Passer de l'autre côté}

Mis en contact avec les profondeurs telluriques, le voyageur se voit ménager une communication avec « l'autre monde », celui des forces redoutables, attirantes et terrifiantes. L'abîme n'est pas simplement un obstacle dans le cours du voyage : il se met à exister pour lui-même, il absorbe le passant dans son vide, lui offrant la révélation d'une plongée dans sa propre intimité. Le sujet s'apparaît comme le passager de son propre être, mais d'un être qui lui demeure inconnu et soudainement creusé.

Mais ce voyageur, s'il passe le l'autre côté, que trouve-t-il ? Paradoxalement, cela n'importe pas toujours, tant l'accent est mis sur l'abîme lui-même. Pourtant, en divisant l'espace, l'abîme structure aussi une topographie symbolique : plus il est profond, inquiétant..., plus la différence des deux côtés doit être forte. Cela se produit, en effet, à chaque fois que les voyageurs passent les Alpes pour se rendre en Italie (par opposition au voyage qui est séjour et station). Nul mieux que Stendhal n'a mis en évidence cette antinomie des deux côtés, dans le récit de sa traversée des Alpes, qu'il représente comme le moment de son entrée dans une nouvelle vie ${ }^{7}$. On est en 1800 , il a dix-sept ans, et va en Italie avec l'armée de Napoléon. Il y découvrira d'un coup la musique et le paysage italiens, la gloire et l'amour... Dans la vision rétrospective de 1836, au moment où il rédige ses souvenirs, il fait de la Suisse un lieu magique ( A Rolle ou à Nyon, je ne sais lequel [...], commença le temps heureux de ma vie "), et du Saint-Bernard l'espace par excellence du franchissement initiatique. Si la montée, le froid, le brouillard, la fatigue, le contexte militaire ont leur importance, l'insistance sur les précipices est frappante, notamment dans les dessins dont il accompagne sa description. Or, 
au passage du col, près de l'Hospice et le long du petit lac, il n'y a pas véritablement de précipice... En tout cas, Horace-Bénédict de Saussure, qui décrit les mêmes lieux, qu'il a parcourus vingt-cinq ans auparavant, n'en mentionne aucun $^{8}$.

Une semblable division de l'espace, un semblable débouché vers la lumière et la vie, marquent le récit que fait Eugène Fromentin d'un de ses voyages en Afrique du Nord. Le réemploi des thèmes romantiques y est patent. Fromentin raconte son hiver de 1848 en Algérie : fuyant la pluie et le froid, il se réfugie au Sahara. Bien que la description strictement topographique apparaisse quelque peu embarrassee, on reconnaît immédiatement les thèmes « alpins » :

El-Kantara - le pont - garde le défilé et pour ainsi dire l'unique porte par où l'on puisse du Tell pénétrer dans le Sahara. Ce passage est une déchirure étroite, qu'on dirait faite de main d'homme, dans une énorme muraille de rochers de trois ou quatre cents pieds d'élévation. Le pont, de construction romaine, est jeté en travers de la coupure. Le pont franchi, et après avoir fait cent pas dans le défilé, vous tombez, par une pente rapide, sur un charmant village, arrosé par un profond cours d'au et perdu dans une forêt de vingt-cinq mille palmiers. Vous êtes dans le Sahara ${ }^{9}$.

La scène est parfaite, du point de vue structurel et du point de vue thématique : deux pays, deux saisons, deux mondes coupés par une fissure et reliés par un pont... Fromentin décrit longuement « le pays céleste du bleu », qu'il valorise par ses qualités climatiques et lumineuses. La force symbolique de la figure du franchissement est si évidente ici qu'elle se soumet toutes les particularités géographiques. Dans cette Afrique saharienne, les thèmes orientaux se superposent à ceux du voyage en Suisse pour mettre en scène comme un coup de theâtre l'opposition du Nord au Sud :

Ce passage inattendu d'une saison à l'autre, l'étrangeté du lieu, la nouveauté des perspectives, tout concourut à en faire comme un lever de rideau splendide, et cette subite apparition de l'Orient par la porte d'or d'El-Kantara m'a laissé pour toujours un souvenir qui tient du merveilleux.

En fait, l'opposition du sombre au lumineux, du froid au chaud, de l'humide au sec, est d'abord structurale et thématique, et peut se déplacer dans les aires géographiques très diverses. Ainsi George Sand, dans Un hiver à Majorque, fait de cette île méditérranéenne une espèce de Suisse où les gouffres jouent leur rôle convenu.

\section{Des interprétations ultérieures}

Il y aurait bien d'autres textes à citer, dans toutes les langues européennes, et de tous les écrivains du XIXe siècle ; bien des tableaux à commenter, des gravures à analyser ${ }^{10}$. La figure du franchissement est un des moments les plus souvent présents et les plus fortement dramatisés dans les récits de voyage romantiques et post-romantiques. Cette figure indéfiniment répétée et variée, la relation trop évidemment spéculaire qui lie le sujet et l'espace, l'inquiétude ou l'espérance de l'autre côté - tout cela pose des questions qu'on pourrait formuler de la manière suivante : si la figure du franchissment construit des représentations récurrentes de 
l'espace parcouru, ressemblantes au-delà des disparités individuelles, géographiques et temporelles, à quoi répond-elle elle-même, par quoi est-elle modélisée ? Et plus particulièrement : pourquoi se représente-t-elle symboliqment comme abîme ? Pourquoi la séparation, frontière ou obstacle, dès qu'il s'agit de la franchir, trouve-t-elle une représentation privilégiée dans les gouffres et les précipices? En général, on traite ce genre de problèmes en montrant que la représentation de la nature s'est modifiée aux XVIIIle et XIXe siècles, passant de la conception d'une nature harmonieuse et géométrique, à une nature plus " primitive ", foisonnante et indomptée. Mais cette esthétique historique du paysage, si elle introduit l'abîme dans un ensemble cohérent, renvoyant à une " mentalité » datée, ne rend pas assez justice à sa puissance symbolique et n'éclaire pas suffisamment le lien central entre le sujet et l'espace parcouru.

A ces questions, on peut chercher réponse chez d'autres écrivains, dans d'autres récits de voyage. Une des caractéristiques des récits romantiques en effet (et du romantisme en général), c'est leur conscience des stéréotypes, du susbstrat citationnel à l'œuvre dans la perception des espaces, et tout particulièrement s'agissant de ces lieux de configuration qu'on rassemble ici sous l'égide d'une poétique du voyage, mais qui constituent aussi bien une dramaturgie des instants nodaux et des décors significatifs. Les romantiques voyagent dans des textes ou dans des images avant de parcourir des pays. Les termes même de pittoresque ou de romantique indiquent ce renvoi à du déjà-vu, déjà-narré, rééprouvé d'autant plus intensément par le voyageur que s'y mêle un sentiment de passé : l'un dit la qualité picturale d'un paysage ou d'une scène, l'autre s'est confondu à ses débuts avec le romanesque. On peut invoquer une sorte de paramnésie pour expliquer ce plaisir particulier du voyageur à percevoir le paysage à travers du déjà-connu, et donc à sentir dans l'impression actuelle sourdre la sensation disparue. On peut faire appel aussi à des considérations socio-historiques et montrer que la multiplication et la diffusion des vues de paysages suisses ont littéralement imprégné les esprits d'images de montagnes. Cette diffusion quasi industrielle s'est accrue durant tout le XIXe siècle, et des écrivains comme Dumas, Hugo ou Gautier en avaient une conscience aiguë puisqu'ils jouent sans cesse des attentes de leurs lecteurs dans la narration de leur voyage. Stendhal lui aussi note cet effet dans sa Vie de Henry Brulard, au moment du passage du Saint-Bernard. Il voit les Alpes en rêvant aux phrases par lesquelles Jean-Jacques Rousseau pourrait les décrire. Il croit être entré à l'hospice, mais n'en est pas sûr : les récits qu'on lui fit de l'intérieur de l'hospice, écrit-il, " produisirent une image qui depuis trente-six ans a pris la place de la réalité " (p. 941 ; souligné par Stendhal). Il en va de même de la descente du col, dont le souvenir réel est effacé par celui d'une gravure de Vernet qu'il a vue plus tard. Ce mouvement de remplacement d'une réalité vue par une image connue ultérieurement n'est pas différent de l'effet pittoresque ou romantique. Au contraire : si des représentations peuvent se subsituer aux choses vues, c'est qu'elles sont aptes à les interpréter, tout comme elles le sont à construire les realia de la perception.

Ainsi les figures du voyage (dans le récit aussi bien que dans l'iconographie) sont des interprétations qui fonctionnent sur plusieurs plans. Elles construisent la connaissance des pays réels perçus par les voyageurs. Elles constituent des réemplois d'images ou de textes antérieurs qu'elles donnent à revoir et à relire, mais aussi qu'elles modifient en les recontextualisant, autrement dit en les intégrant à 
un cadre historique, il le modifie et le déploie symboliquement, il en donne à connaître les enjeux par son acte d'appropriation et par sa propre pratique de représentation.

Voici une description de paysage avec gouffre. Il faut la lire intégralement :

Soudain donc je me trouve au bord d'un précipice, au fond duquel un torrent circule en bouillonnant. Plus encore que du lieu que rien n'amenait, je suis stupéfait du détail, de tous ces détails, du paysage alpestre, avec la vie et l'animation qu'il aurait dans la nature et sans que j'aie eu à m'exciter, à m'enthousiasmer, sans en avoir eu le temps. Sur le champ, de lui-même, il est « lancé ».

Entre cette tranche de pays de montagne si miraculeusement reçue, et un je ne sais plus quoi qui vient d'avoir lieu sur le lieu même de l'expérience, je sais, j'éprouve qu'il doit y avoir un rapport. Qu'était-ce donc ? Me trouvant dans les rochers, $j$ ai peine à revenir dans ma chambre et dans la demi-minute précédente, à contre-courant d'un courant si puissant. Enfin m'y revoici pour inspection, et j'y retrouve - mais oui, c'est cela - l'insolite passage profond et rafraîchissant d'une gorgée d'eau que j'avais avalée et qui descendit en moi profondément. Voilà le rapport. Un instant, je le vois. L'instant d'après, je ne le vois plus, de nouveaux arrivages de pensées, d'images et de poussées ayant intercepté le fragile lien découvert. Revenu aux rochers, niais, mais sachant que j'ai « su $*$, je contemple le cañon redevenu indéchiffrable. La faute en est à la mise en scène du haschich, toujours si réaliste, à ces roches, à ces éboulis, à ce torrent, issus sans doute de ma sensation, mais qui sont d'un ordre tel et d'une profusion spectaculaire telle qu'ils s'opposent à la résurrection de la modeste impression première, laquelle a totalement disparu, ayant fait place à du solide, à du scénique, selon l'évolution commune en cette drogue et qui se fait dans le sens suivant (jamais dans l'autre), du senti vers l'évoqué, du qualificatif vers le substantif, de l'impression vers la chose, de la chose vers le panorama de choses et vers le décor. Ce que je vois pourrait être, en effet, une gravure romantique, mais réifiée soudainement, animée par un presque réel ruisselet qui cascade dans le fond ${ }^{11}$.

Tirée d'un livre dans lequel Michaux consigne ses expériences de drogue, et dont le titre est tout un programme, cette page présente pour nous un extraordinaire intérêt. Elle décrit une vue alpestre, donnée immédiatement comme la substitution d'une image à une réalité d'un autre ordre : en somme, elle part de la représentation pour interroger son origine. De la figure du franchissement, prise dans le moment de la contemplation, tout se retrouve ici, décor, éléments, fascination du sujet, conscience du caractère citationnel. Autant qu'une enquête sur le rapport entre la vue du gouffre et la drogue, cette page mène une interrogation sur le sens du cliché romantique, dès lors qu'il est réinvesti par un sujet dans une situation particulière. Or, cette relation entre ce qui advient au sujet et la vision du précipice, qui constitue une interprétation du gouffre romantique, n'est jamais donnée comme une relation en miroir. Au contraire, la notation insistante du vaet-vient entre les « rochers » et la « chambre », qui représente le questionnement du sens de la scène, montre bien qu'entre le sujet et le précipice se joue un rapport d'extériorisation et d'intériorisation. Ce rapport naît dans le corps (l'eau, la sensation, la profondeur) et dans le langage (l'homophonie gorgée-gorge). Entre l'univers du corps et celui de l'image d'une part, et entre deux signifiants linguistiques d'autre part, s'établit une relation analogique qui peut s'étendre à tous les éléments mis en rapport : c'est le sujet tout entier et sa situation qui relèvent de la 
représentation romantique du gouffre. Celle-ci est le symbole de celui-là, ou plus exactement, elle en est le symbolisé (« l'évoqué, dit Michaux, et plus loin « la chose »), qui devient à son tour symbolisant, représentation et réalité en même temps, où le sujet se reconnaît et se prend.

Dans la découverte-création d'un symbole, il faut donc voir également le réemploi d'une représentation ancienne et une contextualisation nouvelle (ici l'expérience des stupéfiants et autres drogues, si éminemment liés au romantisme par de Quincey et Baudelaire). La symbolique abyssale, dont la fréquence et la puissance expressive et organisatrice pourraient faire penser aux archétypes jungiens, doit être comprise en fait comme un processus dynamique, comme une formation qui a trouvé un jour une configuration opportune et qui, dans une période déterminée et pour une culture déterminée, peut être déployée et interprétcée.

Reste à reprendre la question : pourquoi, à partir de la deuxième moitié du XVIIIe siècle environ, et jusqu'à une époque récente, voit-on se développer si fortement des relations symboliques entre l'homme et la nature ? Jusque-là, les processus symboliques étaient fixés sur les liens entre Dieu et le monde, compris d'une manière à la fois analogique et de parties à totalité. Images de Dieu et éléments du monde, les hommes y trouvaient leur place : selon la formule augustinienne, ils étaient, comme toutes les choses, vestigia trinitatis, des traces de la trinité. Lorsque cet univers de compréhension se brise, une forme essentielle de modélisation, constitutive de l'équipement mental humain, devient vacante ${ }^{12}$. Mis en présence du monde sans la médiation d'une totalité « régulatrice » (qui a pu prendre, un temps, la forme d'un horloger ou d'un géomètre), l'homme reporte directement sur la nature le mouvement de connaissnce symbolique qui transitait auparavant par la divinité. Au lieu de déchiffrer Dieu dans les figures du monde, il va s'y lire lui-même, comprendre le monde comme une projection de lui-même, et par conséquent le méconnaître. Au moment où le voyageur romantique aurait chance de voir la nature et l'homme "tels qu'en eux-mêmes", il les manque, puisqu'il en fait des supports, très vite convenus, d'une exploration de son propre " moi ». Telle est la finalité du voyage romantique : non d'aller à la rencontre d'une altérité, mais de descendre dans les profondeurs d'un moi qui n'a plus d'image transcendante et que la vie sociale oblitère. Ce mouvement de découverte d'un moi plus « profond », Michaux le reporte sur le voyage de la drogue. L'abîme qui s'ouvre ici devant sa conscience et dans son corps, s'il le vit comme un bonheur et un jaillissement, il en connaît aussi les angoisses mieux que personne. La connaissance par les gouffres constitue en fait une part essentielle de son œuvre et qui aide à mieux cerner cette question : qu'est-ce qui est figuré et dramatisé dans le gouffre?

Dans Ecuador, son deuxième livre ${ }^{13}$, Michaux fait de l'Equateur un lieu-moi, un objet et un habitat qu'il repousse et accueille par une méditation poétique qui en sollicite constamment les virtualités symboliques. Michaux lui aussi est l'habitant des abîmes. La drogue, l'écriture, la pensée, l'homme même, il les placera sous cet éclairage équatorien. Mais il accomplit l'intériorisation que les voyageurs romantiques n'avaient pas achevée : il ouvre à l'intérieur de lui-même l'inquiétant néant, le gouffre à peine dissimulé. Si l'Equateur peut devenir un lieu symbolique pour Michaux, c'est qu'une textualité antérieure le configure ; l'écriture de ce lieu éclaire à son tour, rétrospectivement, les modélisations qui gouvernent les figures du voyage. 


\section{Une topographie historique du sujet}

Revenons à notre point de départ. Entre le sujet et le monde s'opèrent des substitutions. L'un devient une partie de l'autre, ou son image, et par ce mouvement de représentation réciproque, une connaissance émerge dans le sujet comme appartenance à son objet. Cette connaissance de l'objet (du gouffre, en l'occurrence) dote celui-ci d'une efficacité symbolique qui lui donne sur le sujet un pouvoir métamorphosant. Le récit de voyage, et plus particulièrement chacune de ses figures, prend naissance au croisement de cet échange. Il organise une succession de compromis symboliques formés par l'ajustement du sujet et du monde. Mais un récit, comme tout texte, ne vient jamais seul, l'écrivain tire de sa langue et des traditions littéraires un trésor et un outillage qui modélise son propre travail, et qu'il adapte à ses besoins. Ainsi, chez un Leiris, l'Abyssinie fait sa place à la thématique orientale, érotisme, déguisements, rites mystérieux et trafics louches compris. Mais de la Porte et du Rempart, de tous les dispositifs d'interdiction, dédales impénétrables ou murs de sérail, si puissants dans les récits romantiques et qui augmentent si remarquablement l'attrait inquiétant des clichés orientaux, Leiris fait un usage nouveau, accordé au monde qu'il vit et à sa disposition subjectale : il met en évidence la frontière et ses imbroglios politicoadministratifs ; et il s'approprie les mystères de l'Abyssinie comme une préfiguration de l'inconscient, du ça archaïque. Le Tibet et l'Equateur proposent aussi l'occasion de réemplois de motifs romantiques, et transposent dans des géographies lointaines les chormos alpestres. La Suisse du XIXe siècle apparaît rétrospectivement comme une sorte d'« Equateur » de l'Europe, quoique dans un contexte historique tout différent ; ou encore comme le lieu d'une découverte mélancolique du moi disparu. Segalen, Michaux ou Leiris transportent aux dimensions de la planète un système de symboles hérités et l'interprètent dans des termes partiellement nouveaux, tout en éclairant ses sens anciens, c'est-à-dire en nous permettant de comprendre à quelles nécessités il a répondu.

Ils explicitent ainsi une sorte de topographie historique du sujet. L'abîme apparaît comme la transposition dans la verticalité d'une limite de type territorial. Il est un symbole chargé de forces contradictoires : il creuse la séparation, la difficulté - voire l'impossiblité - du franchissement ; et en même temps il manifeste la perplexité, et parfois l'angoisse, du sujet devant lui-même, devant une identité que rien n'assure, ni garantie d'en haut, ni trame de ressemblances et de différences. Reprise d'une génération à l'autre, cette représentation s'est vue interprétée peu à peu sous plusieurs perspectives, elle a été en somme approfondie jusqu'à trouver, avec Leiris et Michaux, une intensité et une force qui semblent indépassables, et qui ont sans doute tiré parti des théories psychanalytiques naissantes sur le clivage du moi et sur la mélancolie (théories qui apparaissent elles-mêmes comme des explicitations analytiques et abstraites des thèmes romantiques). La tension entre les valeurs d'interdiction, de transition et de perte de soi, présentes dès les textes « originaires » de Rousseau, est admirablement résumée dans ces réemplois modernes. L'explicitation de la symbolique du gouffre comme limite ou frontière et l'intériorisation achevée de cette symbolique rendent évidente également la valeur de marquage réciproque entre le monde et l'homme. La marque gravée dans le monde et dans le sujet constitue un double stigmate, une sorte de 
tatouage intériorisé par le sujet d'un côté, correspondant de l'autre au tracé d'un fossé dans l'espace naturel. L'établissement d'une topographie symbolique ne serait-elle pas une totémisation du lien entre l'homme et le monde?

On ne peut oublier, d'autre part, que cette topographie accompagne le mouvement d'expansion, puis de reflux, du colonialisme européen, et celui de l'acculturation de zones toujours plus larges de la planète : "Cette terre est rincée de son exotisme », écrit Michaux dans Ecuador. " Nous souffrons mortellement ; de la dimension, de l'avenir de la dimension dont nous sommes privés, maintenant que nous avons fait à satiété le tour de la terre. » L'histoire de l'abîme constate une limite imposée à l'ardeur conquérante et à la raison exploratrice, une sorte d'échec du franchissement. Il n'y a plus d'ailleurs ni d'évasion, thèmes si fréquemment exploités précédemment. De Chateaubriand prenant son baptême des gouffres dans le Nouveau Monde, à Leiris finalement déçu par son séjour abyssin, où plus rien ne lui semble exotique, on est passé d'une France en expansion à une France conduite à reconnaître lentement, par la voix de quelques écrivains, les impasses de son histoire ${ }^{14}$.

\section{NOTES}

1. Ce texte sera repris dans Les Vacances du lundi (1881, posthume). Cité ici d'après Gautier, Impressions de voyage en Suisse, L'Age d'Homme, 1985, p. 185.

2. Voir * La littérature comme interprétation symbolique », dans L'Interprétation des textes, sous la direction de Claude Reichler, Minuit, 1989.

3. Tiré d'une lettre préparée durant le voyage en Suisse que fait Hugo en 1839 (publication posthume dans Alpes et Pyrénées en 1890). Cité ici d'après Victor Hugo, Voyage en Suisse, L'Age d'Homme, 1982, p. 98-99.

4. Publié pour la première fois, comme l'extrait mentionné à la note 1, en 1868 et repris dans

Les Vacances du lundi. Voir T. Gautier, Impressions de voyage en Suisse, éd. cit., p. 171.

5. A. Dumas, Impressions de voyage en Suisse, La Découverte, Maspéro, 1982, t. II, p. 211.

6. Je cite d'après l'édition de Maurice Regard, Pléiade, 1969, t. I. p. 245.

7. V. la Vie de Henry Brulard.

8. Je renverrai au choix de textes publiés par Roger Canac, La Découverte, Maspéro, 1979, sous le titre de Premières ascensions au Mont-Blanc; le passage du Grand-Saint-Bemard est raconté p. 157 et suiv. De Saussure avait publié ses Voyages dans les Alpes de 1779 à 1796. Passionné par la montagne (géologie, climat, faune et flore, population, mythologie...), il ne partage pas la fascination pour l'abîme propre aux romantiques. Mais la célèbre série de gravures dues à $M$. Woscher met en évidence avec insistance les crevasses et les précipices que franchissent les voyageurs dans leur ascension : comme si, dans ce paysage des hauteurs, sublime et désolé, le véritable danger était les trous, comme si les difficultés venaient moins de l'ascension des parois que de la menace de chute dans les abîmes : interprétation du XIXe siècle...

9. Eugène Fromentin, Un été dans le Sahara (première publication 1857); cité d'après Euvres complètes, éd. de Guy Sagnes, Pléiade, 1984, p. 15.

10. Pour une étude globale, voir Le Voyage en Suisse, par Claude Reichler et U. Heidmann Vischer, à paraître.

11. Henri Michaux,Connaissance par les gouffres, Gallimard, 1967, p. 122-124.

12. Voir n. 2.

13. Ecuador. Journal de voyage, 1928 (nouv. éd., rev. et corr., Gallimard, 1969).

14. La présente étude appelle un développement à propos de certains auteurs de voyages modernes abordés dans notre conclusion, notamment Michel Leiris, L'Afrique fantôme (1934) et Victor Segalen, Equipée (1929). Voir mon article * Frontières du monde et territorialité du sujet », dans L'Humanisme en géographie, A. Bailly et R. Scariati éd., Anthropos, 1990. 